\title{
MÖSSBAUER SPECTROSCOPY APPLIEO TO THE OXIDIZED AND SEMI-REDUCEO STATES OF THE IRON-MOLYBDENUM COFACTOR OF NITROGENASE
}

William E. Newton $a^{\star}$, Stephen F. Ghellera, Richard H. Sandsb and W.R. Dunham $b^{*}$

awestern Regional Research Center, USDA-ARS, Albany, CA 94710 and Department of Agronomy and Range Science, University of California, Davis, CA 95616

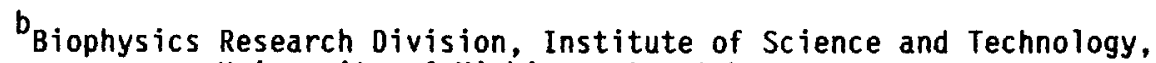
University of Michigan, Ann Arbor, MI 48109

Received June 19, 1989

Mössbauer parameters at $125 \mathrm{~K}$ for both the oxidized and semi-reduced states of FeMoco isolated from the MoFe protein of Azotobacter vinelandii nitrogenase of $\delta / F e=0.32$ and $0.37 \mathrm{~mm} / \mathrm{s}$ and $\Delta E q=\overline{0.84}$ and $0.71 \mathrm{~mm} / \mathrm{s}$, respectively, are reported. FeMoco(ox) fits the Debye model perfectly from 4.2-125K and has a $S=0$ ground state. FeMoco(ox) apparently contains 10-20\% FeMoco(s-r) and vice versa, possibly as a result of the spontaneous oxidation phenomenon. Quantitation of the spectra indicates a Fe:Mo ratio of $5+7: 1$ and the similar quadrupole splittings and isomer shifts suggest a similar environment for all iron atoms. O 1989 Academic Press, Inc.

Feloco extruded from the MoFe protein of nitrogenase has been under active study with respect to its molecular structure, particularly through XAS (1-5), its composition (6-8), and oxidation-reduction properties $(9,10)$, since its original isolation (11). Isolated FeMoco retains its characteristic spectroscopic signatures, only slightly changed from those observed when bound within the protein $(1,2,12,13)$, and is capable of restoring dinitrogen-fixing activity to the FeMoco-deficient, inactive nitrogenase of various mutants of Azotobacter vinelandii $(11,14)$ and Klebsiella pneumoniae (15). Genetic evidence strongly implies that FeMoco is, contains, or is part of the $\mathrm{N}_{2}$-binding site (16). Thus, studies of this simpler entity should give insight into the mechanism of biological dinitrogen reduction.

Although thermally stable in non-aqueous solvents $(6,11,17)$, FeMoco has resisted all attempts to produce $X$-ray diffraction quality crystals. However, XAS at the Mo $K$-edge shows three iron, three sulfur and three oxygen nearest neighbors $(1,2)$. How the remaining atoms are arranged and exactly how many

* To whom correspondence should be addressed.

Abbreviations Used: FeMoco, iron-molybdenum cofactor; MoFe protein, molybdenumiron protein of nitrogenase: XAS, X-ray absorption spectroscopy: NMF. N-methylformamide; EPR, electron paramagnetic resonance; $(0 x)$, oxidized; $(s-r)$, semi-reduced; (red), reduced; ENDOR, electron-nuclear double resonance; EFG, electric field gradient. 
of each are required to complete the Felloco unit are unknown. FeMoco in NMF exhibits two redox couples, which interconvert the EPR-active $S=3 / 2$ Feloco(s-r), the state produced by excess dithionite, with both a more oxidized state, FeMoco(ox), and a more reduced state, FeMoco(red) (9). The $(0 x)-t o-(s-r)$ change is a reversible, one-electron process $(9,10)$.

In contrast to the intensive Mössbauer (18-20) investigation of the holoMoFe protein, much of which impacts directly on FeMoco bound within the protein, one report only mentions the Mössbauer spectrum of isolated FeMoco(s-r) (12). The lack of comparative information for bound versus isolated FeMoco in both $(o x)$ and $(s-r)$ states, plus our desire to: (i) quantitate the Fe content of FeMoco; (ii) determine the spin state of FeMoco(ox); (iii) determine where the added $e^{-}$is accommodated in FeMoco(s-r); and (iv) gain insight into the symmetry of this heterometallic cluster, led to the Mössbauer study reported herein, which emphasizes the FeMoco(ox) state.

\section{MATERIALS ANO METHODS}

The Mössbauer spectrometer used has been described previously $(21,22)$. It permits very precise measurement of the source lineshape (21), which allows us to deconvolve the data and perform the logarithmic conversion from transmission to absorption spectra $(22,23)$. However, it is not possible to present simple absorption spectra, when there are non-trivial magnetic terms present in the spin Hamiltonian (21). Therefore, because some spectra are taken under high applied magnectic fields, we show all spectra in transmission mode for consistency.

57 Fe-labelled FeMoco was isolated from Azotobacter vinelandii strain oP, which was grown in batch culture in acid-washed glass vessels essentially as described previously (24) except that the Burk nitrogen-free medium contained $57 \mathrm{Fe}$ (New England Nuclear, $96.5 \%$ ) at only $20 \%$ of the usual iron concentration. Isolation and analys is of the $57 \mathrm{Fe}$-labelled nitrogenase proteins were as reported earlier (24). The $57 \mathrm{Fe}$-enriched MoFe protein of specific activity 1877 nmol $\mathrm{C}_{2} \mathrm{H}_{4}$ formed min ${ }^{-1}$ (mg protein) ${ }^{-1}$ contained 24.5 iron atoms per molecule $(25)$.

57 Fe-enriched FeMoco was released from this protein into NMF and concentrated as previously described (6). Its specific activity was $197 \mathrm{nmol}^{\mathrm{C}_{2} \mathrm{H}_{4}}$ formed $\min ^{-1}$ (ng. atom Mo) ${ }^{-1}$. The Mo and Fe concentrations were $0.214 \mathrm{mM}$ and $1.055 \mathrm{mM}$, respectively, $(25,26)$ giving $\mathrm{Fe}:$ Mo of $4.9: 1$. Aliquots were transferred to a Mössbauer cuvette $(200 \mu \mathrm{L})$ and an EPR tube $(300 \mu \mathrm{L})$ in a Vacuum Atmospheres anaerobic glove box, sealed, removed from the glove box and frozen in liquid $N_{2}$. EPR spectroscopy (19) at lOK showed a $S=3 / 2$ signal with $g$ values typical of FeMoco(s-r). Mössbauer spectroscopy (see Results and Discussion section) indicated some FeMoco(ox), so the sample was thawed anaerobical$l y$, dithionite $(10 \mu \mathrm{L}$ of $0.2 \mathrm{M}$ solution) added, and, after $10 \mathrm{~min}$, refrozen in liquid $N_{2}$. The remainder of the sample was allowed to oxidize spontaneously (9) and Znaerobically at $-80^{\circ} \mathrm{C}$ over a period of four weeks. Mössbauer and EPR aliquots were then removed and frozen as before. Unenriched 0.734 and 1.054 $\mathrm{mM}$ (in Mo) FeMoco samples in both the $(\mathrm{ox})$ and $(s-r)$ states were prepared and investigated similarly. The Fe: Mo ratio for both samples was $5.8: 1$ with specific activities of $170 \mathrm{nmol}^{\mathrm{C}_{2} \mathrm{H}_{4} \text { formed min-1 (ng. atom Mo) }}{ }^{-1}$. The EPR samples of FeMoco(ox) were EPR-silent. EPR experiments were also performed directly on the samples in the Mössbauer cuvettes using a Varian "wide mouth" cylindrical cavity operating in the TE-013 mode. The FeMoco(s-r) samples gave the expected $S=3 / 2$ EPR spectrum, but the experiments failed to detect the 
FeMoco(s-r) molecules in the FeMoco(ox) Mössbauer samples because the low filling factor of the cavity generated insufficient signal-to-noise.

\section{RESULTS ANO DISCUSSION}

Fig. I shows a stackplot of FeMoco(ox) at zero applied magnetic field and at temperatures from 4.2 to 175K. A major quadrupole doublet is visible at all temperatures. In addition, three other signals are observed. The first, a high-spin ferrous component, has been observed previously (12). Its

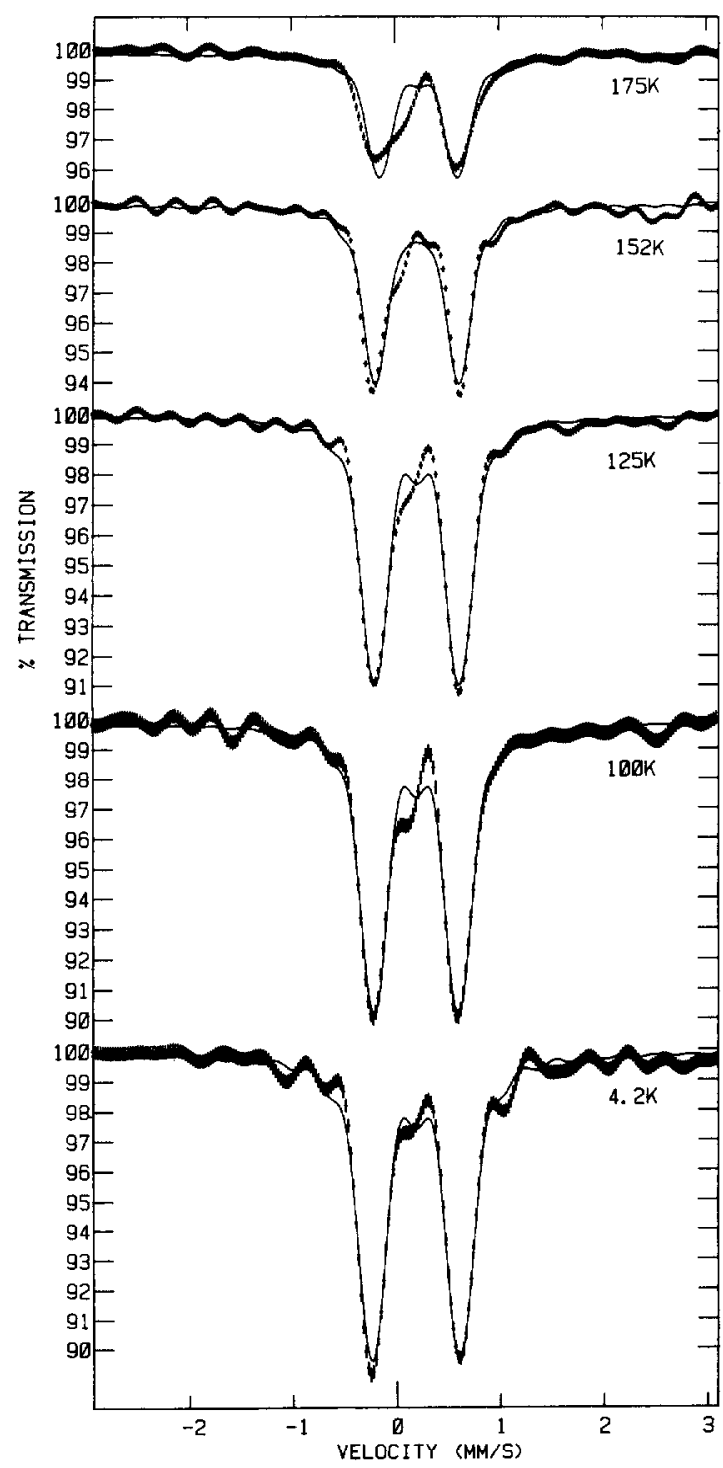

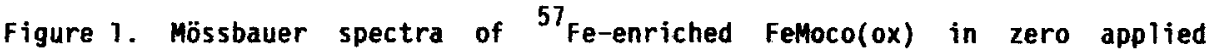
magnetic field as a function of temperature. The solid lines are single site fits with the parameters shown in Table 1. 
right-hand line is sometimes visible at $2.6 \mathrm{~mm} / \mathrm{s}$. The second signal is the quadrupole doublet $(\delta / \mathrm{Fe}=0.55 \mathrm{~mm} / \mathrm{s}$ and $\Lambda \mathrm{Eq}=0.88 \mathrm{~mm} / \mathrm{s})$ that appears as shoulders on the right-hand side of the major doublet. We have very carefully quantitated these signals as maximally $3 \%$ and $6 \%$ of total iron, respectively; much less than the $12.5 \%$ of intensity minimally expected for an integral component of intact FeMoco. Both then are assigned to low-concentration contaminants. The third signal ( $73 \%)$ arises from FeMoco(s-r). Its concentration was estimated from the decrease in intensity of the main quadrupole doublet as temperature is lowered (Table 1), because the FeMoco(s-r) quadrupole doublet lies within the lineshape of FeMoco(ox).

The amounts of iron listed in Table 1 include an assumed Debye temperature of 188K, which was chosen to perfect the model between 100 and $125 \mathrm{~K}$. If the sum of the intensity of all the lines is used, then the fit of the Debye model to intensity is perfect over 4.2-125K. There is, however, a very narrow temperature range, near $125 \mathrm{~K}$, where the feMoco(ox) resonances are faithfully represented by quadruple doublets. Above $150 \mathrm{~K}$, the intensity decreases due to the onset of the liquid state of NMF. The individual iron atoms of isolated Felloco, therefore, do not have Debye-Waller factors with different temperature dependencies as reported for the ' $P$ ' clusters of the MoFe protein (19). The isomer shift variation with temperature (lable 1 ) is probably due to the 2nd-order relativistic Doppler shift, but no such correction is applied. A similar temperature dependence of isomer shift is found with spectra of both mode 1 compounds (prismanes and cubanes) and nitrogenase.

Table 1. Best Fit Parameters of the Two-Line Model for FeMoco(ox $)^{a, b}$

\begin{tabular}{cccc}
\hline$T(K)$ & $\begin{array}{c}\delta / \mathrm{Fe} \\
(\mathrm{mm} / \mathrm{s})\end{array}$ & $\begin{array}{c}\Delta \mathrm{Eq} \\
(\mathrm{mm} / \mathrm{s})\end{array}$ & \#umoles ${ }^{57 \mathrm{Fe}}$ \\
\hline 175 & 0.32 & 0.76 & 0.12 \\
152 & 0.33 & 0.81 & 0.21 \\
125 & 0.33 & 0.82 & 0.22 \\
100 & 0.34 & 0.82 & 0.22 \\
4.2 & 0.35 & 0.85 & 0.19 \\
\hline
\end{tabular}

ancertainty values are $0.01,0.02$ and 0.01 for $\delta / \mathrm{Fe}, \Delta \mathrm{Eq}$ and \# $\mu \mathrm{moles}{ }^{57} \mathrm{Fe}$, respectively (22).

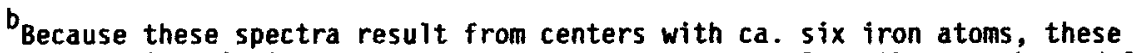
single site fitting parameters represent average values that can be modeled under the simulation linewidth of $0.27 \mathrm{~mm} / \mathrm{s}$ (FWHW). 
To determine if FeMoco(ox) is diamagnetic at low temperature (Fig. 2), a very intense magnetic field ( 5 Tesla) was applied to the sample at $4.2 \mathrm{~K}$ to ensure that the expected zeeman splitting of an integer spin system would be greater than KT and the expected zero-field splittings. For non-zero spin, magnetic splitting from the ground state of the spin multiplet is expected, but the only magnetic splitting observed is due to the minor contribution from FeMoco(s-r). This result proves that isolated FeMoco(ox) has a $S=0$ ground state, the same as when bound to the protein (27).

The spectra of Felloco(s-r) at high temperature and at low temperature and low applied field are shown in Figure 3 . Under each set of conditions, two spectra are reported. The initial point spectra indicated the presence of FeHoco(ox), which was subsequently reduced by added dithionite as shown by the diminished intensity of the central quadrupole doublet and the increased intensity of the outer lines. Based on estimated intensity of the central
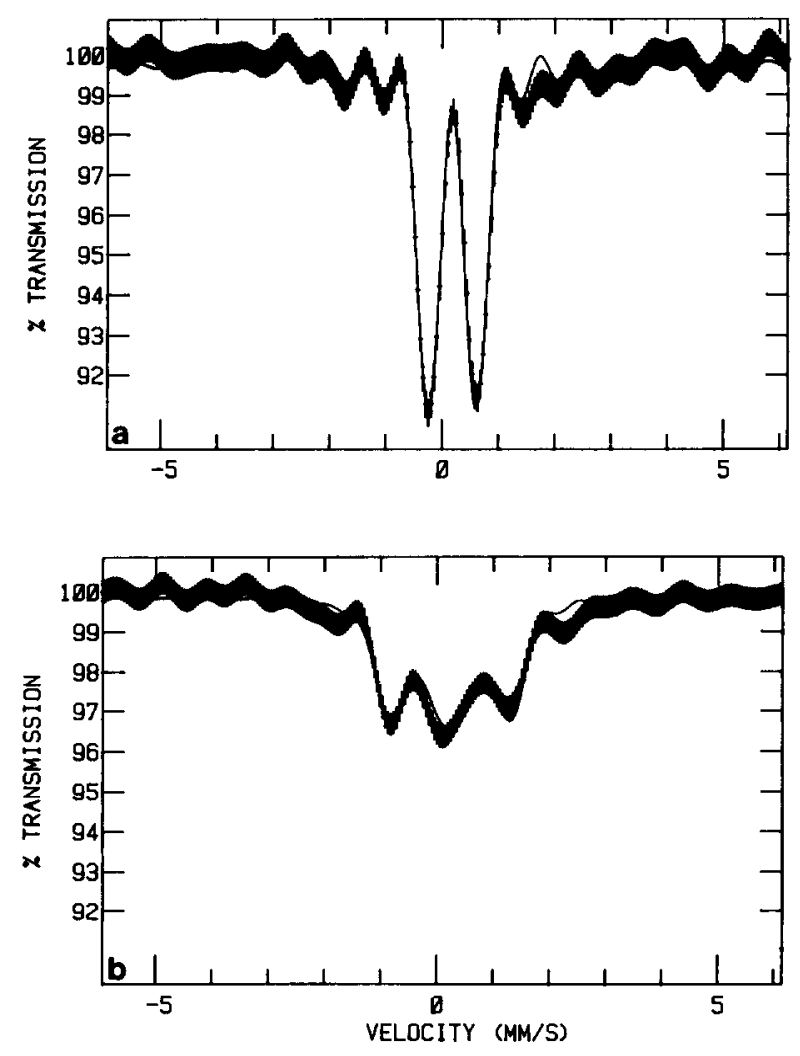

Figure 2. Low temperature (4.2K) Mössbauer spectra of ${ }^{57}$ Fe-enriched FeMoco(ox) at 0.06 Tesla (a) and at 5 Tesla (b) applied magnetic field. The solid lines are computer simulations with $\triangle E q=0.85$ $\mathrm{mm} / \mathrm{s}$, asymmetry parameter $n=0$, the appropriate applied fields and with linewidths of $0.27 \mathrm{~mm} / \mathrm{s}$ (a) or $0.45 \mathrm{~mm} / \mathrm{s}$ (b). 

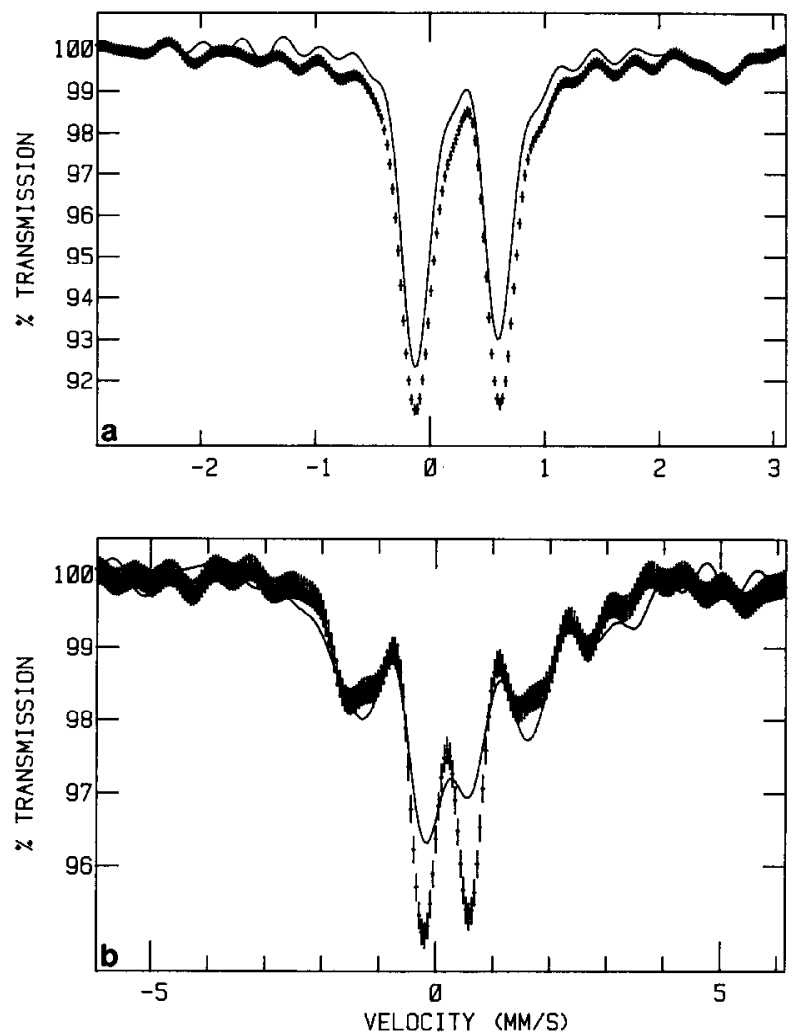

Figure 3. Mössbauer spectra of 57 Fe-enriched FeMoco(s-r) at $125 \mathrm{~K}$ in the absence of an applied field (a) and at $4.2 \mathrm{~K}$ in a 0.06 Tes la applied magnetic field (b); (…) initial spectra, (-) after additional aqueous dithionite. The solid line spectra have slightly reduced intensity due to dilution.

quadrupole pair, a change from ca. $30 \%$ oxidized to ca. $20 \%$ oxidized occurred, assuming identical Debye temperatures for Feloco(ox) and $(s-r)$. These low-temperature spectra are entirely consistent with that reported previously (12), which also contained ca. $20 \%$ of its intensity as a then uncharacterized quadrupole doublet that we now suggest is Feloco(ox). Thus, the spectra of isolated FeMoco(ox) appear to contain a contribution from FeMoco(s-r) and vice versa, possibly due to the spontaneous oxidation phenomenon $(9,28)$. If this situation is germane to the MoFe protein, then interpretation of the Mössbauer spectra of many protein samples could be severely compromised.

By using the low temperature spectra to quantitate the percent reduction of the sample, the best fit of the high temperature components in Figs. Ic and 3a can be calculated after subtraction of the minor component. Iteration of this fitting procedure gives the parameters for pure FeMoco(ox) and $(s-r)$ (Table 2), which show that the change in isomer shift accompanying a one-elec- 
Table 2. Mössbauer Parameters of FeMoco and Chemical Models

\begin{tabular}{|c|c|c|c|c|c|}
\hline Compound & $T(K)$ & $\begin{array}{c}\delta / \mathrm{Fe} \\
(\mathrm{mm} / \mathrm{s})\end{array}$ & $\begin{array}{c}\Delta E q \\
(m n / s)\end{array}$ & $\begin{array}{c}\text { pmoles } \\
57 \\
7_{\mathrm{Fe}}\end{array}$ & Ref. \\
\hline FeMoco $(0 x)^{a}$ & 125 & 0.32 & 0.84 & 0.22 & This work \\
\hline FeMoco $(s-r)^{a}$ & 125 & 0.37 & 0.71 & 0.17 & This work \\
\hline FeMoco(s-r) & 90 & 0.37 & 0.75 & --- & (12) \\
\hline$\left(\mathrm{Ph}_{4} \mathrm{P}\right)_{2} \mathrm{Fe}_{6} \mathrm{~S}_{6} \mathrm{Cl} \mathrm{l}_{6}$ & 125 & 0.43 & 0.62 & --- & (34) \\
\hline$\left.\left(E t_{4} N\right)_{3} \mathrm{Fe}_{6} \mathrm{~S}_{6} \mathrm{Cl}\right]_{6}$ & 125 & 0.50 & 1.09 & --- & (35) \\
\hline$\left(\mathrm{Et}_{4} \mathrm{~N}\right)_{2} \mathrm{Fe}_{4} \mathrm{~S}_{4}\left(\mathrm{SCH}_{2} \mathrm{Ph}\right)_{4}$ & 77 & 0.36 & 1.10 & -- & (36) \\
\hline$\left(\mathrm{Et}_{4} \mathrm{~N}_{3}\right)_{3} \mathrm{Fe}_{4} \mathrm{~S}_{4}\left(\mathrm{SCH}_{2} \mathrm{Ph}\right)_{4}$ & 77 & 0.44 & 1.00 & $-\cdots$ & (37) \\
\hline$\left(\mathrm{Bu}_{4} \mathrm{~N}\right)_{3} \mathrm{Mo}_{2} \mathrm{Fe}_{6} \mathrm{~S}_{8}(\mathrm{SPh})_{9}$ & 130 & $0.28^{b}$ & $1.12^{b}$ & --- & (38) \\
\hline$\left(\mathrm{Et}_{4} \mathrm{~N}_{5}\right)_{5} \mathrm{Mo}_{2} \mathrm{Fe}_{6} \mathrm{~S}_{8}(\mathrm{SPh})_{9}$ & 130 & $0.39^{b}$ & $1.28^{b}$ & ---- & (38) \\
\hline
\end{tabular}

a These measurements and the related simulations assume that both lines of the quadrupole pairs have a linewidth of $0.27 \mathrm{~mm} / \mathrm{s}$ (FWHM).

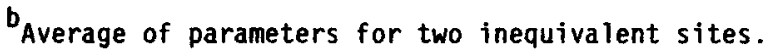

tron change is $0.05 \mathrm{~mm} / \mathrm{s}$. The analogous shift for cubane and prismane models is about $0.08 \mathrm{~mm} / \mathrm{s}(p=0.01)$. As all are iron/sulfur clusters with similar numbers of iron atoms, the reducing electron appears to be delocalized less onto the iron atoms of FeMoco. Certainly, orbitals with a large molybdenum component are good candidates for accommodating the additional charge density but recent XAS work is inconsistent with this suggestion, leaving orbitals with a large sulfur component as the additional acceptors $(3,30)$.

An accurate determination of Felloco's Fe-to-Mo ratio is needed. Because our spectrometer is quantitative, iron content is easily determined which,

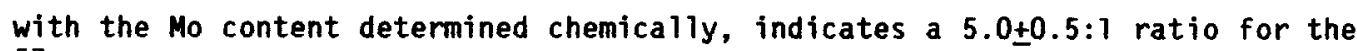
57 Fe-enriched samples. Four other FeMoco samples isolated with naturally abundant ${ }^{57} \mathrm{Fe}$ contents had a ratio of $5.25+0.25: 1$. All contain ca. $9 \%$ of total iron as impurities. Assuming only iron in the main doublet is in Felloco, plus all the molybdenum, a Fe:Mo ratio of $4.3: 1$ results. If the minor quadrupole doublet, which is responsible for the $6 \%$ iron impurity, also contains molybdenum in a $1: 1$ ratio, the Fe:Mo ratio approximates $6: 1$. obviously, the Fe:Mo ratio depends very strongly on both Mo content and Fe-containing impurities and can only be approximated as $5 \pm 1: 1$. This value encompasses a previous estimate (12). The accuracy of Mössbauer spectroscopic iron determinations depends critically on the validity of the model 
used. For the MoFe protein (18), the intensity of a particular spectral line is used to quantitate others. The complexity of the spectra shown herein hint that this spectroscopic model may lack the accuracy necessary to determine Felloco's Fe:Mo ratio. ENDOR measurements (31) are free of some of these interpretation difficulties but unfortunately, this technique has not yet been applied to isolated FeMoco. Even so, the five distinct ${ }^{57} \mathrm{Fe}$ resonances reported for bound FeMoco(s-r) confirm a Fe:Mo ratio of at least $5: 1$.

The narrow linewidths of our quadrupole doublets suggest equivalent environments for iron in isolated FeMoco. In contrast, based on an analysis of the ENDOR ${ }^{57} \mathrm{Fe}$ magnetic hyperfine tensor components, low symmetry is suggested for bound FeMoco (31). However, the ENDOR work ignored the importance in spin coupled systems of the projection operator term $(32,33)$, which describes the orientation of component spins relative to the resultant. For bound FeMoco, three iron atoms have negative and two have positive hyperfine couplings (31), indicating complex spin coupling among an unknown number of components. This situation makes it impossible to determine symmetry by comparing the magnitude of hyperfine coupling tensors. However, the single site fit to our data (Fig. 2D) implies that the EFG tensors could be identical for all FeMoco iron atoms. To the extent that the EFG tensor connotes ligand symmetry, such identity is a strong constraint on site geometry - much stronger than spin-coupling details implied by the magnitude of hyperfine coupling tensors. Further, the quadrupole doublet in the $4.2 \mathrm{~K}$ spectrum of FeMoco(s-r) (Fig. 3b) could be due to an iron site of FeMoco(s-r) with the projection operator term equal to zero rather than to residual FeMoco(ox). In this case, the Fe-to-Mo ratio of FeMoco would increase to $6 \pm 1: 1$. We would, therefore, point the Felloco model builders toward a structure that is symmetric with respect to the iron atoms (at least five and possibly six) and oxidized with respect to the irons in the oxidized (2-) prismane.

\section{ACKNOWLEDGMENTS}

We thank 6.D. Watt, 2.-C. Wong (assistance with ${ }^{57}$ Fe-MoFe protein isolation), D.L. Jacobs (assays) and the National Institutes of Health (Grants DK-37255 to WEN and GM-32785 to RHS).

\section{REFERENCES}

1. Conradson, S.D., Burgess, B.K., Newton, W.E., Mortenson, L.E. and Hodgson, K.0. (1987) J. Amer. Chem. Soc. 109, 7507-7515.

2. Conradson, S.D. Burgess, B.K.. Newton, W.E., Hodgson, K.O. and McDonald, J.W. Rubinson, J.F., Gheller, S.F., Mortenson, L.E., Adams, M.W.W., Mascharak, P.K., Armstrong, W.A., and Holm, R.H. (1985) J. Amer. Chem. Soc. 107, 7935-7940. 
3. Hedman, B., Frank, P., Gheller, S.F., Roe, A.L., Newton, W.E., and Hodgson, K.0. (1988) J. Amer. Chem. Soc. 110, 3798-3805.

4. Antonio, M.R., Teo, B.-K., Orme-Johnson, W.H., Nelson, M.J., Groh, S.E., Lindahl, P.A., Kauzlarich, S.M. and Averill, B.A. (7982) J. Amer. Chem. Soc. $104,4703-4705$.

5. Arber, J.M., Flood, A.C., Garner, C.D., Gorma1, C.A., Hasnain, S.S., and Smith, B.E. (1988) Biochem. J. 252, 421-425.

6. Yang, S.-S., Pan, W.-H., Friesen, D.G., Burgess, B.K., Corbin, J.L., Stiefel, E.I., and Newton, W.E. (1982) J. Biol. Chem. 257, 8042-8048.

7. Nelson, M.J., Levy, M.A., and Orme-Johnson, W.H. (1983) Proc. Nat1. Acad. SCi. USA $80,147-150$.

8. Lowe, D.J., Thorneley, R.N.F., and Smith, B.E. (1985) In Metalloproteins, Part I: Metal Proteins with Redox Roles (P.H. Harrison, Ed.), pp. 207-249. Macmillan Press, London.

9. Schultz, F.A., Gheller, S.F., Burgess, B.K., Lough, S., and Newton, W.E. (1985) 3. Amer. Soc. 107, 5364-5368.

10. Schultz, F.A., Gheller, S.F., and Newton, W.E. (1988) Biochem. Biophys. Res. Commn. 152, 629-635.

11. Shah, V.K. and Brill, W.J. (1977) Proc. Nat. Acad. Sci. USA 74, 3249-3253.

12. Rawlings, J., Shah, V.K., Chisnell, J.R., Brill, W.J., Zimmermann, R., Munck, E., and Orme Johnson, W.H. (1978) J. Biol. Chem. 253-1001-1004.

13. Burgess, B.K., Stiefe1, E.I., and Newton, W.E. (1980) J. Biol. Chem. 255, $353-356$.

14. Brigle, K.E., Weiss, M.C., Newton, W.E., and Dean, D.R. (1987) J. Bacterio1. 169, 1547-1553.

15. Hawkes, T.R. and Smith, B.E. (1983) Biochem. J. 209, 43-50.

16. Hawkes, T.R., McLean, P.A., and Smith, B.E. (1984) Biochem. J. 217, $317-321$.

17. Lough, S.M., Jacobs, D.L., Lyons, D.M., Watt, G.D., and McDonald, J.W. (1986) Biochem. Biophys. Res. Commn. 139, 740-746.

18. Smith, B.E., O'Donnell, M.J., Lang, G. and Spartalian, K. (1980) Biochem. J. 191, 449-455 and refs. therein.

19. Mclean, P.A., Papaefthymiou, V., Orme-Johnson, W.H., and Munck, E. (1987) J. Biol. Chem. 262, 12900-12903 and refs. therein.

20. Dunham, W.R., Hagen, W.R., Braaksma, A., Grande, H.J., and Haaker, J. (1985) Eur. J. Biochem. 146, 497-501.

21. W.F. Filter (1983) High Resolution ${ }^{57} \mathrm{Fe}$ Mössbauer Spectroscopy, Ph.D. Thesis, The University of Michigan.

22. W.R. Dunham, C.T. Wu, R.M. Polichar, R.H. Sands and L.J. Harding (1977) Nuc 1. Inst. Meth. 145, 537-553.

23. The procedure of M.C. Dibar-Ure and P.A. Flinn (1971), in Mössbauer Effect Methodology (I.J. Grewerman, C.W. Seidel, and D.W. Dieterly, Eds.), Vol. 9, pp. 204-233. Plenum Press, New York, is unnacceptable unless corrected for Gaussian convolution effects and source lineshape at which point it becomes equivalent to ours.

24. Burgess, B.K., Jacobs, D.L., and Stiefel, E.I. (1980) Biochim. Biophys. Acta $614,196-209$.

25. Dieh1, J., Smith, G.F., McBride, L., and Cryberg, R. (1965). In The Iron Reagents, pp. 1-64. The G. Frederick Smith Chemical Co., Columbus, OH.

26. Clark, L.J. and Axley, J.H. (1955) Anal. Chem. 27, 2000-2003.

27. Zimmermann, R., Munck, E., Bril1, W.J., Shah, V.K., Henz1, M.T., Rawlings, J. and Orme-Johnson. W.H. (1978) Biochim. Biophys. Acta 537, 185-207.

28. McKenna, C.E., Menard, R., Dao, C.J., Stephens, P.J., and McKenna, M.C. (1988) In Nitrogen Fixation: One Hundred Years After ( $H$. Bothe, F.J. de Bruijn and W.E. Newton, Eds. ) p. 131. Fischer Verlag, Stuttgart, F.R.G.

29. Dunham, W.R., Hagen, W.R., Braaksma, A., Haaker, J., Gheller, S., Newton, W.E., and Smith, B. (1985) In Nitrogen Fixation Research Progress (H.J. Evans, P.J. Bottomley and W.E. Newton, Eds.), pp. 597-596. Martinus Nijhoff, Dordrecht, The Netherlands.

30. Mijller A., Hellman, W. Schimanski, V., Jostes, R., and Newton, W.E. (7983) Z. Naturforsch. 38b, 528-529. 
31. True, A.E., Nelson, M.J., Venters, R.A., Orme-Johnson, W.H., and Hoffman, B.M. (1988) J. Amer. Chem. Soc. 110, 1935-1943 and refs. therein.

32. Griffith, J. S. (1971) Mol. Phys. 21, 141-143.

33. Dunham, W. R., Sands, R. H., Shaw, R. W. and Beinert, H. (1983) Biochim. Biophys. Acta 748, 73-85.

34. Coucouvanis, D., Kanatzidis, M.G., Dunham, W.R., and Hagen, W.R. (1984) J. Amer. Chem. Soc. 106, 7998-7999.

35. Kanatzidis, M.G., Hagen, W.R., Dunham, W.R., Lester, R.K. and Coucouvanis, 0.(1985) J. Amer. Chem. Soc. 107, 953-961.

36. Holm, R.H., Averill, B.A., Herskovitz, T., Franke1, R.B., Gray, H.B., Si iman, 0., and Grunthamer, F.J. (1974) J. Amer. Chem. Soc. 96, 2644-2646.

37. Lane, R.W., Wedd, A.G., Gillum, W.O. Laskowski, E.J., Holm, R.H., Frankel, R.B., and Papaefthymiou, G.C. (1977) J. Amer. Chem. Soc. 99, 2350-2352.

38. Christou, G., Mascharak, P.K., Armstrong, W.H., Papaefthymiou, G.C., Frankel, R.B., and Holm, R.H. (1982) J. Amer. Chem. Soc. 104, 2820-2831. 